\title{
ESPÉCIES DE Candida spp. EM ISOLADOS CLÍNICOS E SUSCETIBILIDADE A ANTIFUNGICOS DE USO HOSPITALAR
}

\section{Vanusa Aparecida Althaus}

Farmacêutica, Pós-graduanda em Análises Clínicas na Universidade Comunitária Regional de Chapecó (UNOCHAPECÓ), SC, Brasil.

\section{Alissara Regginato}

Farmacêutica, Pós-graduanda em Farmacologia Clínica na Universidade Comunitária Regional de Chapecó (UNOCHAPECÓ), SC, Brasil

\section{Vanessa Bossetti}

Farmacêutica pela Universidade Comunitária Regional de Chapecó (UNOCHAPECÓ), SC, Brasil (UNOCHAPECÓ)

\section{Juliana Cristina Schmidt}

Farmacêutica Industrial e Bioquímica (UFPR); Mestre em Bioquímica (UFPR); Doutora em Biologia Celular e Molecular (UFPR); Docente da Área de Ciências da Saúde e da Pós-graduação em Diagnóstico Clínico Laboratorial e Farmacologia na Universidade Comunitária Regional de Chapecó (UNOCHAPECÓ), SC, Brasil.

E-mail: jcs@unochapeco.edu.br
RESUMO: A levedura Candida spp., pertencente à microbiota normal, pode causar graves infecções, principalmente em pacientes no ambiente hospitalar. Os variados graus de patogenicidade e sensibilidade a antifúngicos apresentados pelas diversas espécies, combinados ao aumento da frequência de infecções por Candida spp. e às altas taxas de morbimortalidade, apontam a importância da identificação das espécies, assim como a realização de testes de suscetibilidade para o sucesso do tratamento de candidíases. Neste estudo foram identificadas espécies de Candida em isolados clínicos de pacientes atendidos em um hospital da região oeste de Santa Catarina, Brasil, utilizando o método CHROMagar Candida, bem como avaliados seus respectivos perfis de suscetibilidade a antifúngicos usados no âmbito hospitalar utilizando o método de disco-difusão em ágar. Das 43 amostras analisadas, a maior prevalência da candidíase foi encontrada em pacientes com idade entre 51 e 70 anos e do gênero masculino. A maioria do isolados foi obtida de amostras de urina $(\mathrm{n}=25)$ e da Unidade de Terapia Intensiva $(\mathrm{n}=22)$. As espécies mais frequentes foram $C$. albicans $(\mathrm{n}=24)$ e $C$. glabrata $(\mathrm{n}=13)$. A maioria dos isolados foi sensível à anfotericina B $(86,05 \%)$ e a minoria $(13,95 \%)$ sensível ao fluconazol. O setor de UTI, as amostras de urina e as culturas identificadas como $C$. albicans apresentaram vários isolados resistentes a todos os antifúngicos testados. O elevado número de isolados resistentes aos antifúngicos azólicos, provenientes principalmente de pacientes de UTI, indicam a importância do antifungigrama para a escolha do antifúngico e da dose mais adequados no tratamento de candidíases em pacientes críticos.

PALAVRAS-CHAVE: Candidíase; Infecção Hospitalar; Testes de Sensibilidade Microbiana.

\section{Candida spp. IN CLINICAL ISOLATES AND SUSCEPTIBILITY Y TO ANTIFUNGALS AGENTS IN HOSPITALS}

\begin{abstract}
The yeast Candida spp. belongs to the normal microbiota and may cause severe infections, mainly in hospitalized patients. The various degrees of pathogenicity and sensitiveness to antifungal agents by the several species, plus increase in the frequency of infections by Candida spp. and high morbo-mortality rates, underscore the importance of identifying the species and undertaking susceptibility tests for the treatment of candidiasis. Candida species were identified by the CHROMagar Candida method, in clinical isolates from patients who attended a hospital in the western region of the state of Santa Catarina, Brazil. Susceptibility profiles to antifungal agents used in hospital were evaluated by the agar disc-diffusion method. The greatest prevalence of candidiasis in 43 samples occurred in male patients within the 51-70 age brackets. Most isolates were obtained
\end{abstract}


from urine samples $(n=25)$ and from ICU $(n=22)$. The more frequent species comprised $C$. albicans $(\mathrm{n}=24)$ and $C$. glabrata $(\mathrm{n}=13)$. Most isolates were sensitive to amphotericin B (86.05\%) and the lowest (13.95\%) were sensitive to fluconazol. Urine samples in ICU and culture identified as $C$. albicans had several isolated resistance to all antifungal agents tested. The high number of isolates resistant to azolic antifungal agents, mainly from ICU patients, indicates the importance of antifungal testing to select the most suitable antifungal and dose for the treatment of candidiasis in critical patients.

KEY WORDS: Candidiasis; Hospital Infection; Microbial susceptibility Testing.

\section{INTRODUÇÃO}

Embora as leveduras do gênero Candida sejam habitantes comensais de diversas partes do corpo, colonizando o trato gastrointestinal em $80 \%$ da população adulta saudável e a vagina em 20 a 30\% das mulheres, nos hospitais correspondem a cerca de $80 \%$ das infecções fúngicas documentadas (AVRELLA; GOULART, 2008; ÁLVARES; SVLDZLNSKI; CONSOLARO, 2007).

A transformação da levedura de comensal a agente infeccioso ocorre principalmente em ambiente hospitalar, com o aumento do número de procedimentos invasivos quebrando barreiras de proteção natural, o uso intensivo de antimicrobianos de amplo espectro e o maior número de pessoas debilitadas e suscetíveis a microrganismos oportunistas (BARBEDO; SGARBI, 2010). Alguns dos fatores mais comuns para a colonização por leveduras são imunossupressão por diferentes causas, neutropenia, desnutrição e quimioterapia antineoplásica, o uso prolongado de cateteres, queimaduras e cirurgias extensas. Devem ser incluídas como causas das variações imunológicas as alterações fisiológicas provocadas em decorrência da prematuridade ou do envelhecimento (COLOMBO; GUIMARÃES, 2003; ÁLVARES; SVLDZLNSKI; CONSOLARO, 2007).

As infecções fúngicas de origem hospitalar passaram a apresentar grande importância nos últimos anos, pelo seu aumento progressivo e por suas elevadas taxas de morbidade e mortalidade (FIDEL; VAZQUEZ; SOBEL, 1999). Além disso, a resistência aos agentes antifúngicos entre as espécies de Candida spp. tem sido um problema crescente, tornando-se necessária a realização de testes de suscetibilidade nos isolados clínicos. A identificação prévia da espécie de Candida e a determinação da suscetibilidade in vitro têm sido recomendadas em alguns casos, como na candidíase de orofaringe de pacientes infectados com HIV, micoses sistêmicas e vaginites recorrentes (GIOLO; SVIDZINSKI, 2010). Em relação aos aspectos epidemiológicos, a identificação específica de leveduras até o nível de espécie é uma etapa fundamental para monitorar taxas de infecção hospitalar, determinar a etiologia da infecção e detectar novos agentes da doença (MALUCHE; SANTOS, 2008).

A Candida albicans é a espécie mais frequentemente descrita em casos de infecções hospitalares em diversos países (COLOMBO; GUIMARÃES, 2003). Contudo, observa-se um aumento da ocorrência de infecções sistêmicas por espécies não-albicans, que diferem na produção de fatores de virulência e na sensibilidade aos antifúngicos. Desse modo, a identificação da espécie causadora da infecção e a avaliação de sua suscetibilidade aos antifúngicos comumente utilizados podem contribuir para um tratamento mais efetivo da infecção, reduzindo as taxas de morbidade e mortalidade de pacientes em nível hospitalar (BARBEDO; SGARBI, 2010).

Dessa maneira, este estudo pretende contribuir para o conhecimento da epidemiologia das infecções causadas por Candida spp. em pacientes atendidos em hospital, por meio da identificação da espécie causadora da infecção, bem como da verificação do seu respectivo perfil de sensibilidade a antifúngicos comumente utilizados em âmbito hospitalar.

\section{METODOLOGIA}

\subsection{POPULAÇÃO E AMOSTRAGEM}

Para este estudo foram utilizados isolados clínicos obtidos de pacientes atendidos em um hospital da região oeste de Santa Catarina (Chapecó, Santa Catarina, Brasil) no período entre setembro de 2013 a maio de 2014, previamente isolados pelo laboratório de 
análises clínicas conveniado ao hospital. Também foram consultadas as requisições de exame para obtenção das seguintes informações: gênero e idade do paciente, tipo de amostra enviada para análise microbiológica e setor do hospital. As amostras incluídas neste estudo foram todas aquelas previamente isoladas pelo laboratório e identificadas como Candida spp. Os isolados foram semeados em ágar Sabouraud suplementado com cloranfenicol e transportados até o laboratório de Biotecnologia Farmacêutica da Universidade Comunitária da Região de Chapecó (UNOCHAPECO) para realização das análises.

\subsection{IDENTIFICAÇÃO DAS ESPÉCIES DE Candida spp.}

Os isolados cultivados em ágar Sabouraud foram subcultivados em placas contendo meio cromogênico CHROMagar Candida (Microbiology-Plast Labor, Rio de Janeiro, Brasil), realizando a semeadura pela técnica de esgotamento e a incubação em estufa a $32^{\circ} \mathrm{C}$ por 48 horas. O meio cromogênico CHROMagar Candida é utilizado para identificação de espécies de Candida baseado na pigmentação expressa pelas colônias quando cultivadas em temperatura e tempo ideal para crescimento. Após esse período foram analisadas a morfologia e pigmentação do cultivo, de acordo com o descrito por Araujo et al. (2005) e comparando com os controles positivos fornecidos pelo laboratório de Micologia da Unochapecó: C. albicans (ATCC 24433) exibe uma coloração verde; $C$. tropicalis (ATCC 750), azul; C. krusei (ATCC 6258), coloração rósea e com aspecto rugoso e bordas irregulares; $C$. parapsilosis (ATCC 90018), rósea e aspecto liso; e a C. glabrata (ATCC 2001), arroxeada/ilás. Quando duas espécies distintas foram observadas no mesmo cultivo, foi realizado novo subcultivo em meio cromogênico CHROMagar Candida para isolar e identificar as possíveis espécies.

\subsection{AVALIAÇÃO DO PERFIL DE SUSCETIBILIDADE A AN- TIFÚNGICOS}

O perfil de suscetibilidade dos isolados de Candida spp. a diferentes agentes antifúngicos foi realizado pelo método de disco difusão em ágar Miller Hinton, de acordo com o documento M44-A (CLSI, 2004) e seguindo as instruções do fabricante. Nos testes foram utilizados discos contendo os seguintes antifúngicos: fluconazol $(50 \mathrm{mg})$, miconazol $(50 \mathrm{mg})$, cetoconazol $(50 \mathrm{mg})$, flucitosina $(1 \mu \mathrm{g})$ e voriconazol $(1 \mu \mathrm{g})$ (BioRad, Marnes La Coquette, France). Os parâmetros de resistência, sensibilidade e sensibilidade dose dependente foram definidos a partir da medição do diâmetro do halo de inibição de crescimento ao redor dos discos, seguindo as orientações do fabricante conforme descrito na Tabela 1 .

Tabela 1. Critérios utilizados para interpretação da sensibilidade aos antifúngicos, de acordo com o manual do fabricante (BioRad).

\begin{tabular}{ccccc}
\hline Antifúngico testado & $\begin{array}{c}\text { Concentração do } \\
\text { disco }\end{array}$ & Sensível & $\begin{array}{c}\text { Sensível dose- } \\
\text { dependente }\end{array}$ & Resistente \\
\hline Anfotericina B & $100 \mathrm{ug}$ & $>10 \mathrm{~mm}$ & $=10 \mathrm{~mm}$ & $\leq 10 \mathrm{~mm}$ \\
Cetoconazol & $50 \mathrm{ug}$ & $>20 \mathrm{~mm}$ & $10-20 \mathrm{~mm}$ & $<10 \mathrm{~mm}$ \\
Fluconazol & $25 \mathrm{ug}$ & $>19 \mathrm{~mm}$ & $15-18 \mathrm{~mm}$ & $<14 \mathrm{~mm}$ \\
Flucitosina & $1 \mathrm{ug}$ & $\geq 20 \mathrm{~mm}$ & $10-20 \mathrm{~mm}$ & $<10 \mathrm{~mm}$ \\
Voriconazol & $1 \mathrm{ug}$ & $>17 \mathrm{~mm}$ & $14-16 \mathrm{~mm}$ & $<13 \mathrm{~mm}$ \\
\hline
\end{tabular}

Fonte: Bio-Rad Laboratories.

\subsection{ASPECTOS ÉTICOS}

O estudo foi aprovado pelo Comitê de Ética em Pesquisa da UNOCHAPECO, sob protocolos $\mathrm{n}^{0}$ 119/13 e $122 / 13$.

\section{RESULTADOS}

Foram analisados isolados de Candida spp. obtidos de 43 amostras clínicas de pacientes atendidos no hospital durante o período da pesquisa. Deste total, $58 \%(\mathrm{n}=25)$ eram provenientes de indivíduos do gênero masculino e $42 \%(n=18)$ do gênero feminino. A 
distribuição das amostras de acordo com a idade mostrou que a maioria $(44,1 \% ; n=19)$ foi obtida de pacientes com idade entre 51 e 70 anos, com idade média de 60,5 anos.

A análise das espécies de Candida pelo método CHROMagar permitiu identificar 46 espécies nas 43 amostras analisadas, uma vez que em três amostras foram identificadas duas espécies. Foram caracterizados 24 isolados como C. albicans, 13 isolados como C. glabrata, 2 isolados como C. tropicalis, 2 isolados como C. krusei e 5 isolados foram caracterizados como outras espécies diferentes das anteriores (Figura 1).

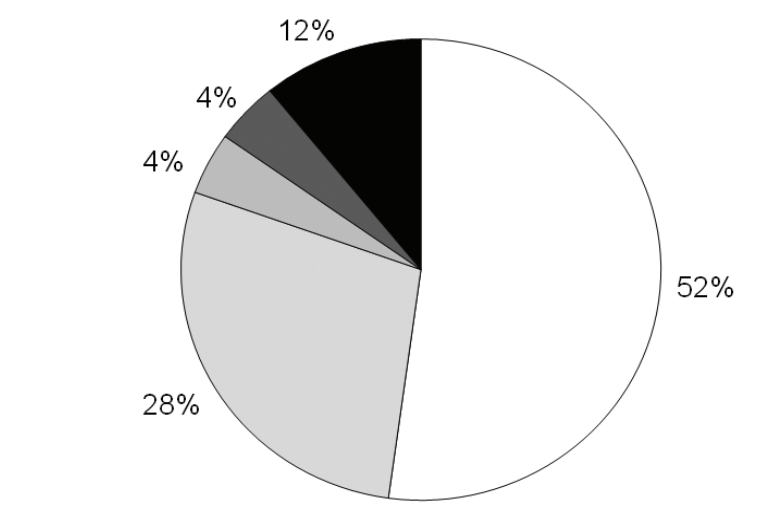

$\square$ C. albicans $\square$ C. glabrata $\square$ C. tropicalis $\square$ C. krusei $\square$ Outras

Figura 1. Distribuição geral de espécies de Candida identificadas nas amostras Fonte: Dados da pesquisa.

O setor do hospital que apresentou maior número de isolados clínicos positivos para Candida spp. foi a Unidade de Terapia Intensiva (UTI) $(51 \%$; $=22)$, seguido pela clínica médica com $23 \%(n=10)$ e a oncologia com $9 \%(n=4)$ de isolados. Os setores de quimioterapia, hemodiálise e centro cirúrgico apresentaram $5 \%(n=2)$ de isolados cada um, e neurologia, $2 \%(n=1)$ de isolados.

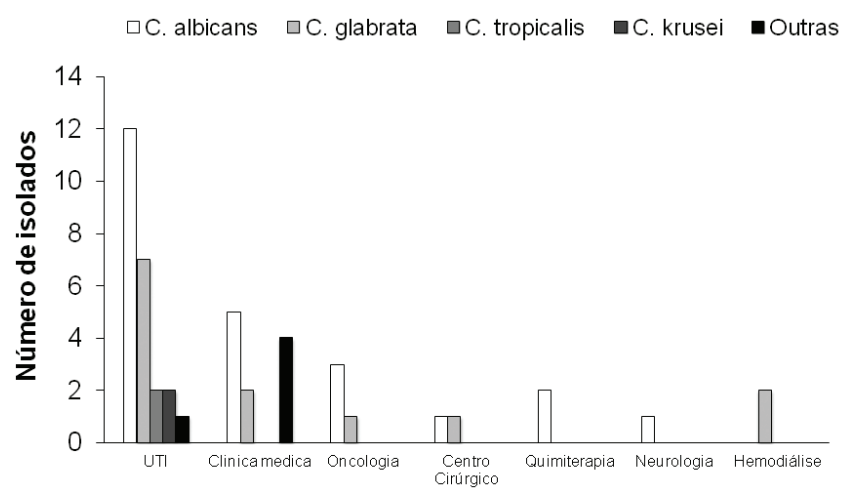

Figura 2. Setores de serviço hospitalar e espécies de Candida identificadas

Fonte: Dados da pesquisa.
Com base nas espécies identificadas nos diversos setores do hospital (Figura 2), observa-se que o setor de UTI apresentou isolados com, pelo menos, um representante de cada espécie identificada neste estudo, além de amostras caracterizadas como outras espécies. As amostras provenientes dos setores de oncologia e centro cirúrgico apresentaram isolados somente de duas espécies, C. albicans e C. glabrata, e as dos setores de quimioterapia e neurologia, somente $C$. albicans. Também se notou que dos cinco isolados de C. krusei, quatro eram provenientes da clínica médica. Já o setor de hemodiálise apresentou somente isolados de C. glabrata.

Em relação ao tipo de amostra clínica analisada verificou-se a predominância de Candida spp. isolada em urina $(58,2 \% ; n=25)$, seguida de aspirado traqueal com 16,3\% ( $n=7)$, sangue com 9,4\% ( $=4)$, lavado brônquico e fezes ambos com 6,9\% $(n=3)$ e secreção de prótese com 2,3\% $(\mathrm{n}=1)$.

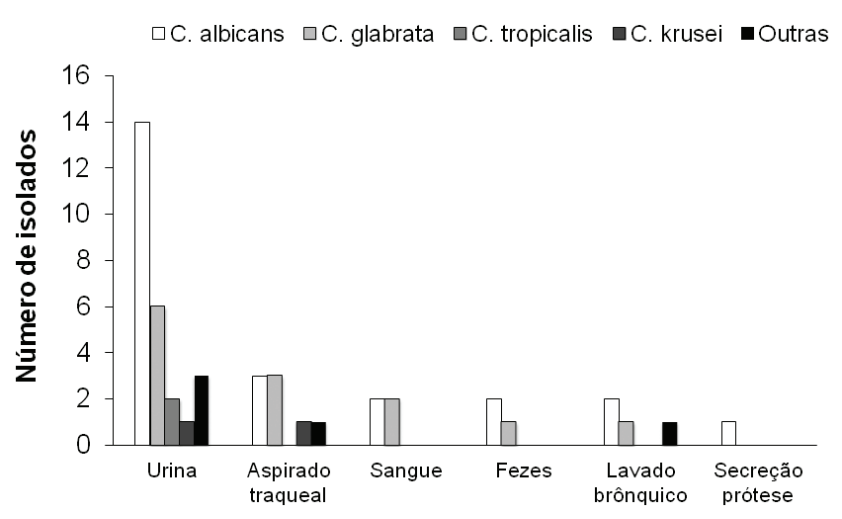

Figura 3. Tipo de amostra e espécies de Candida identificadas Fonte: Dados da pesquisa.

Dentre as espécies identificadas (Figura 3), a $C$. albicans foi a mais frequentemente isolada de amostras de urina e presente em todas as outras amostras clínicas analisadas. Já a espécie C. glabrata não foi encontrada em amostra de secreção de prótese; a C. krusei foi isolada de urina e aspirado traqueal e a $C$. tropicalis foi encontrada somente em amostras de urina.

Vale destacar ainda, que durante a pesquisa, um paciente atendido no setor de quimioterapia do hospital apresentou infecção por Candida em momentos distintos. Nos dois episódios a levedura isolada foi da espécie $C$. albicans, inicialmente em cultura de urina e depois em hemocultura. Outro fato observado com a identificação das espécies foi que alguns isolados se revelaram com 
mais de uma coloração, indicando mais de uma espécie por amostra clínica. Esse fato pôde ser constatado e confirmado após novo subcultivo das espécies no meio cromogênico, sendo possível então isolar as espécies. As amostras analisadas foram lavado brônquico de um paciente da clínica médica, e aspirado traqueal e urina de pacientes distintos da UTI.

O perfil de suscetibilidade das espécies identificadas foi avaliado frente a antifúngicos comumente utilizados no âmbito hospitalar (Figura 4) e revelou que grande parte dos isolados de Candida spp. apresentou sensibilidade a anfotericina B (86,05\%), flucitosina (76,74\%) e voriconazol (74,42\%). Contudo, observouse que $53,49 \%$ e $48,83 \%$ dos isolados foram classificados como sensíveis dose-dependente ou resistentes a cetoconazol e a fluconazol, respectivamente.

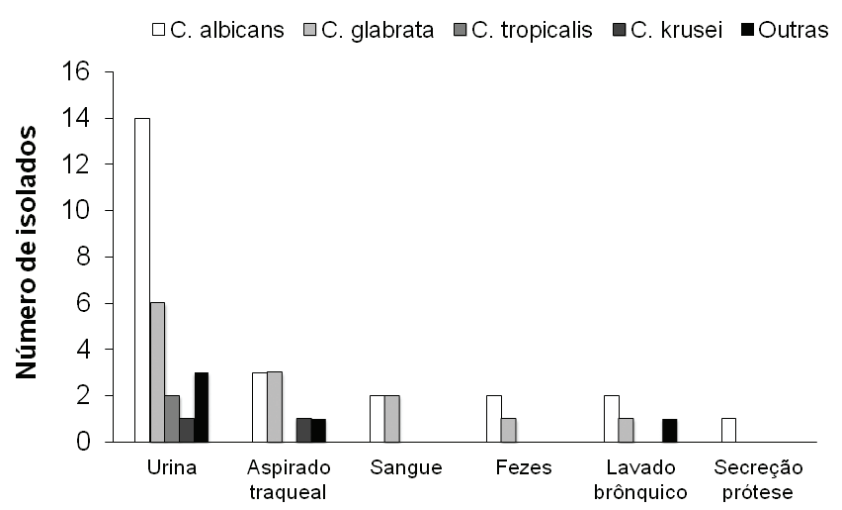

Figura 4. Perfil de suscetibilidade dos isolados de Candida spp. a agentes antifúngicos Fonte: Dados da pesquisa.
$\mathrm{Na}$ avaliação da sensibilidade das leveduras isoladas a partir de diferentes amostras clínicas (Tabela 2), obtidas de pacientes de vários setores do hospital (Tabela 3), verificou-se que as amostras de centro cirúrgico, clínica médica, neurologia e oncologia não apresentaram resistência, assim como as leveduras isoladas de lavado brônquico e sangue. Entretanto, observou-se que o setor de UTI apresentou amostras resistentes aos cinco antifúngicos testados e, dentre estas, 14 (63,6\%) amostras foram sensíveis dose-dependente ou resistentes a fluconazol. Um resultado semelhante foi observado com outro antifúngico azólico, o cetoconazol. As amostras de urina também apresentaram perfil semelhante, com $\mathrm{n}=11(44 \%)$ e $\mathrm{n}=13$ (52\%) das amostras sensíveis dosedependente ou resistentes a fluconazol e cetoconazol, respectivamente.

Tabela 2. Classificação do tipo de amostra de acordo com os perfis de sensibilidade aos diferentes antifúngicos testados

(continua)

\begin{tabular}{|c|c|c|c|c|}
\hline \multirow[b]{2}{*}{ Tipo de amostra clínica } & \multirow[b]{2}{*}{ Agente antifúngico } & \multicolumn{3}{|c|}{ erfil de susceptibilidade in vitro* } \\
\hline & & $\mathbf{S}$ & SDD & $\mathbf{R}$ \\
\hline \multirow{5}{*}{ Urina $(n=25)$} & Anfotericina B & 24 & 1 & 0 \\
\hline & Flucitosina & 22 & 2 & 1 \\
\hline & Cetoconazol & 12 & 9 & 4 \\
\hline & Fluconazol & 14 & 7 & 4 \\
\hline & Voriconazol & 20 & 4 & 1 \\
\hline \multirow{5}{*}{ Aspirado traqueal $(n=7)$} & Anfotericina B & 6 & 1 & 0 \\
\hline & Flucitosina & 5 & 2 & 0 \\
\hline & Cetoconazol & 3 & 3 & 1 \\
\hline & Fluconazol & 3 & 4 & 0 \\
\hline & Voriconazol & 5 & 2 & 0 \\
\hline
\end{tabular}




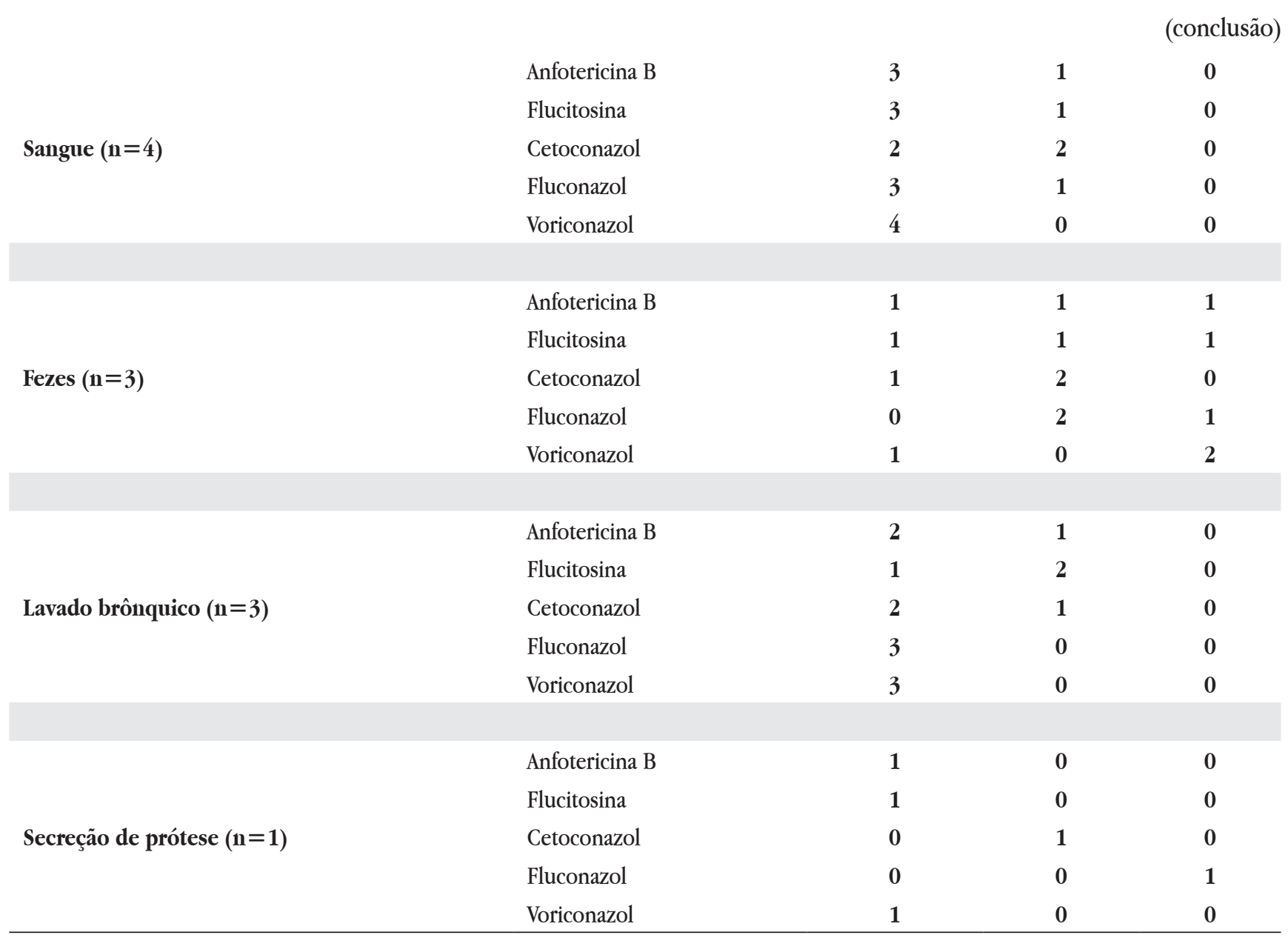

*S: suscetível; SDD: suscetível dose-dependente; R: resistente.

Tabela 3. Classificação dos perfis de sensibilidade aos diferentes antifúngicos testados de acordo com setor hospitalar

(continua)

\begin{tabular}{|c|c|c|c|c|}
\hline \multirow[b]{2}{*}{ Tipo de amostra clínica } & \multirow[b]{2}{*}{ Agente antifúngico } & \multicolumn{3}{|c|}{ rfil de susceptibilidade in vitro* } \\
\hline & & $S$ & SDD & $\mathbf{R}$ \\
\hline \multirow{5}{*}{ UTI $(n=22)$} & Anfotericina B & 19 & 2 & 1 \\
\hline & Flucitosina & 16 & 4 & 2 \\
\hline & Cetoconazol & 8 & 10 & 4 \\
\hline & Fluconazol & 8 & 11 & 3 \\
\hline & Voriconazol & 14 & 4 & 4 \\
\hline \multirow{5}{*}{ Clínica médica $(\mathrm{n}=10)$} & Anfotericina B & 10 & 0 & 0 \\
\hline & Flucitosina & 9 & 1 & 0 \\
\hline & Cetoconazol & 7 & 3 & 0 \\
\hline & Fluconazol & 8 & 2 & 0 \\
\hline & Voriconazol & 9 & 1 & 0 \\
\hline \multirow{5}{*}{ Oncologia $(n=4)$} & Anfotericina B & 1 & 3 & 0 \\
\hline & Flucitosina & 1 & 3 & 0 \\
\hline & Cetoconazol & 2 & 2 & 0 \\
\hline & Fluconazol & 3 & 0 & 1 \\
\hline & Voriconazol & 3 & 0 & 1 \\
\hline
\end{tabular}




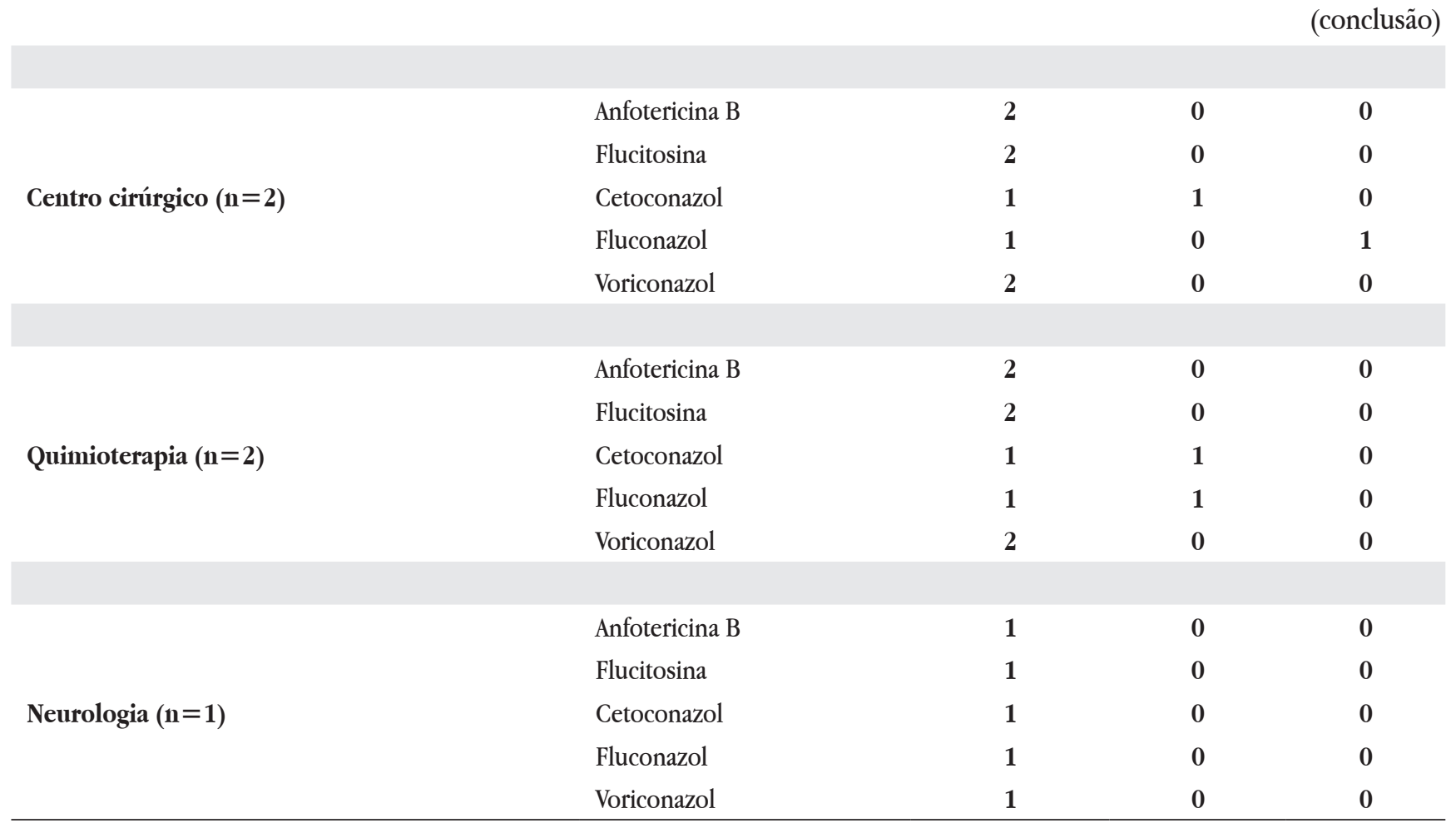

*S: suscetível; SDD: suscetível dose-dependente; R: resistente

Na Tabela 4 são mostradas as espécies de Candida diferente de C. glabrata, que apresentaram resistência identificadas e seus respectivos perfis de sensibilidade a aos azóis, mas não à anfotericina $\mathrm{B}$ e à flucitosina. As antifúngicos. Nesta tabela não foram considerados os dados provenientes das três amostras que apresentavam mais de uma espécie. Vários isolados de C. albicans espécies que não apresentaram isolados resistentes foram C. troplicalis e outras espécies de Candida não apresentaram resistência a todos os antifúngicos testados,

Tabela 4. Sensibilidade in vitro de espécies de Candida a agentes antifúngicos

(continua)

\begin{tabular}{llccc}
\hline & & Perfil de susceptibilidade in vitro* & R \\
\hline Tipo de amostra clínica & Agente antifúngico & S & SDD & R \\
\hline \multirow{4}{*}{ Candida albicans $(\mathbf{n}=\mathbf{2 1})$} & Anfotericina B & 18 & 2 & 1 \\
& Flucitosina & 15 & 4 & 2 \\
& Cetoconazol & 10 & 9 & 2 \\
& Fluconazol & 11 & 5 & 5 \\
& Voriconazol & 15 & 3 & 3 \\
& Anfotericina B & & & \\
& Flucitosina & 10 & 2 & 0 \\
& Cetoconazol & 10 & 2 & 0 \\
& Fluconazol & 6 & 5 & 1 \\
& Voriconazol & 6 & 5 & 1 \\
& & 10 & 1 & 1 \\
& Anfotericina B & & & \\
& Flucitosina & 1 & 0 & 0 \\
& Cetoconazol & 1 & 0 & 0 \\
& Fluconazol & 0 & 1 & 0 \\
& Voriconazol & 1 & 0 & 0 \\
& & 0 & 1 & 0
\end{tabular}




\begin{tabular}{llllc} 
& & & (conclusão) \\
& Anfotericina B & $\mathbf{2}$ & $\mathbf{0}$ & $\mathbf{0}$ \\
Candida krusei $(\mathbf{n}=\mathbf{2})$ & Flucitosina & $\mathbf{2}$ & $\mathbf{0}$ & $\mathbf{0}$ \\
& Cetoconazol & $\mathbf{1}$ & $\mathbf{0}$ & $\mathbf{0}$ \\
& Fluconazol & $\mathbf{0}$ & $\mathbf{2}$ & $\mathbf{0}$ \\
& Voriconazol & $\mathbf{2}$ & $\mathbf{0}$ & $\mathbf{0}$ \\
& & & & \\
& Anfotericina B & $\mathbf{3}$ & $\mathbf{1}$ & $\mathbf{0}$ \\
Outras espécies $(\mathbf{n}=4)$ & Flucitosina & $\mathbf{3}$ & $\mathbf{1}$ & $\mathbf{0}$ \\
& Cetoconazol & $\mathbf{2}$ & $\mathbf{2}$ & $\mathbf{0}$ \\
& Fluconazol & $\mathbf{3}$ & $\mathbf{1}$ & $\mathbf{0}$ \\
& Voriconazol & $\mathbf{3}$ & $\mathbf{1}$ & $\mathbf{0}$ \\
\hline
\end{tabular}

*S: suscetível; SDD: suscetível dose-dependente; R: resistente

\section{DISCUSSÃO}

O gênero Candida se encontra muito bem adaptado ao corpo humano e o coloniza sem produzir sinais de enfermidade. Esta relação de comensal é dependente da integridade do tecido do hospedeiro, da microbiota normal assim como do sistema imune. Por conseguinte, o fungo expressa de forma equilibrada a sua capacidade de aderência e de produção de enzimas e toxinas (SANTANA; RIBEIRO NAVES, 2010; ROSSI et al., 2011). Contudo, a frequência de infecções por espécies do gênero Candida tem aumentado, tornando a candidíase uma infecção muito comum em pacientes hospitalizados. Dentre vários fatores já relacionados que podem contribuir para esse quadro, também pode-se destacar outros como condicionantes para a infecção fúngica, como diabetes mellitus, uso prolongado de antibióticos, AIDS (Síndrome da Imunodeficiência Adquirida), leucemia, queimaduras, transplantes, tratamento quimioterápico e uso de sondas (AVRELLA; GOULART, 2008).

Nos resultados obtidos neste estudo observouse que a maioria do isolados de Candida spp. foi proveniente de UTI $(n=22)$. O aumento acentuado de casos de candidemia, principalmente em doentes de UTI, decorre do aumento do número de fatores de risco, como a diminuição das defesas imunitárias dos doentes, tornando estes pacientes os principais sujeitos à infecção por Candida spp (PEREIRA, 2010; COLOMBO; GUIMARÃES, 2003). Silva (2010) também relata outros fatores predisponentes que podem levar o paciente a uma candidíase, como a granulocitopenia, a disfunção celular (sobretudo de linfócito T), a deficiência humoral congênita ou adquirida, a esplenectomia e a deficiência mecânica (especialmente pela presença de cateteres). Tais fatores tornam-se determinantes para infecções adquiridas por via endógena, resultantes da defesa imunitária comprometida desses pacientes (MARTINS-DINIZetal., 2005). Entretanto, deve-se também considerar as infecções adquiridas por via exógena de várias origens, tais como mãos dos trabalhadores da área da saúde, biomateriais e fontes inanimadas ambientais (OLIVEIRA, 2007). Um estudo realizado revelou que a maioria dos funcionários de um hospital localizada no setor da UTI possuía colonização por Candida spp. nas mãos e que a não ou má higienização correta a cada procedimento e o atendimento a mais de um paciente torna a transmissão cruzada um problema sério (TAMURA, 2007).

A maioria do isolados obtida neste estudo foi proveniente de amostras de urina $(n=25)$, semelhante ao observado por outros autores que apontaram as leveduras do gênero Candida como as principais responsáveis pelas micoses urinárias, ocupando $0 \quad 4^{\circ}$ lugar nas infecções nosocomiais do trato urinário no meio hospitalar (PEREIRA, 2010; NEUFELD, 2009). Mudanças no trato urinário, incluindo anomalias anatômicas, redução do fluxo e alterações na composição da urina são fatores fundamentais para que os fungos se proliferem de maneira a tornar-se um patógeno oportunista (OLIVEIRA, 2007). Sabe-se que sondagem vesical, assim como uso prolongado de cateteres, cirurgias prévias (transplantados), utilização de antibioticoterapia, entre outros, são fatores que favorecem o desenvolvimento 
de candidúria. Embora na maioria dos casos apareça de forma assintomática, a candidúria pode ser o início de processos infecciosos mais graves no rim, causando graves lesões com elevadas taxas de morbidade e mortalidade, implicando inclusive na disseminação hematogênica das leveduras (OLIVEIRA, 2007; FRANÇA; RIBEIRO; QUEIROZ-TELLES, 2008; NEUFELD, 2009).

Dentre as espécies identificadas neste estudo, as mais frequentes foram C. albicans (52\%) e C. glabrata (28\%). A C. albicans é a espécie mais frequentemente descrita em casos de infecções superficiais e invasivas em diversos sítios anatômicos em diversos países (MALUCHE; SANTOS, 2008). Quando ocorre a invasão ao hospedeiro a $C$. albicans leva as células do epitélio a realizarem "internalização celular", mecanismo considerado como fundamental para o crescimento e colonização da Candida no organismo do hospedeiro (ÁLVARES; SVLDZLNSKI; CONSOLARO, 2007; ROSSI et al., 2011). Deve-se destacar também que quase metade dos isolados foi identificada como espécies não-albicans. Esta frequência expressiva de infecções sistêmicas por espécies não-albicans, que diferem na produção de fatores de virulência e na sensibilidade aos antifúngicos, também vem sendo descrita com maior frequência (RÖRIG; COLACITE; ABEGG, 2009; SANTANA; RIBEIRO; MENEZES, 2013). Dessa maneira, a identificação da espécie causadora da infecção e a avaliação de sua suscetibilidade aos antifúngicos comumente utilizados podem contribuir para um tratamento mais efetivo da infecção, reduzindo as taxas de morbidade e mortalidade de pacientes em nível hospitalar (ALVARES; SVLDZLNSKI; CONSOLARO, 2007).

Com relação à suscetibilidade a agentes antifúngicos comumente utilizados em hospital, verificouse que a maioria dos isolados foi sensível à anfotericina $\mathrm{B}$ $(86,05 \%)$ e a minoria $(13,95 \%)$ sensível ao fluconazol. O alto índice de sensibilidade dose-dependente e resistência frente ao fluconazol podem ser explicados pelo uso indiscriminado da medicação, uma vez que a resistência ao fluconazol pode chegar a $45 \%$ entre os indivíduos que receberam terapia prévia, tornando-os mais suscetíveis a apresentar candidemia (ZARDO; MEZZARI, 2004).

Além disso, observou-se que aproximadamente metade dos isolados de C. albicans e C. glabrata apresentou sensibilidade dose-dependente ou resistência aos azólicos testados. Embora a $C$. albicans seja naturalmente sensível aos fármacos antifúngicos de uso sistêmico, relatos de resistência adquirida a azólicos são conhecidos em pacientes que usam por tempo prolongado este tipo de medicamento (BARBEDO; SGARBI, 2010; FRANÇA; RIBEIRO; QUEIROZ-TELLES, 2008).

As infecções provocadas por $C$. glabrata também merecem destaque, pois são difíceis de tratar e muitas vezes são resistentes a muitos agentes antifúngicos azólicos (FIDEL; VAZQUEZ; SOBEL, 1999). Estima-se que em pacientes com fungemia por $C$. glabrata a taxa de mortalidade é em torno de $50 \%$ em pacientes com câncer e 100\% quando em associação a complicações de transplante de medula óssea (BARBEDO; SGARBI, 2010).

Vale ressaltar ainda que vários isolados do setor de UTI, das amostras de urina e das culturas identificadas como C. albicans apresentaram resistência a todos os antifúngicos testados neste estudo. Oliveira (2007) discute que apesar de a profilaxia antifúngica ter efetividade comprovada em pacientes neutropênicos de alto risco, a mesma em pacientes críticos internados em UTI ainda é assunto de muita controvérsia, pois em seu estudo ressalta que a introdução profilática antifúngica, utilizada às vezes de forma equivocada, com o uso do fluconazol, não traz muitos benefícios em termos de sobrevida em pacientes admitidos em UTI, levando talvez à seleção de espécies não-albicans resistentes ao fluconazol.

As razões pelas quais existam altas taxas de infecções por fungos do gênero Candida nos hospitais ainda não são bem claras, mas acredita-se que a grande maioria das infecções seja ainda causada por via endógena. Contudo, é possível que outras vias de contaminação também contribuam de maneira muito significativa, como os diversos padrões de atenção com os doentes graves, número de profissionais de saúde por paciente, qualificação dos profissionais de saúde, reutilização de materiais descartáveis e adesão a práticas padrão de controle de infecção hospitalar (HINRICHSEN, 2008). Soma-se a isto o aumento da resistência a antifúngicos que alerta para a necessidade do desenvolvimento de estratégias que evitem a sua 
disseminação entre os fungos, como já ocorreu com as bactérias (MENEZES; MENDES; CUNHA, 2009). Por este motivo os médicos estão sendo mais criteriosos no momento das prescrições terapêuticas iniciais (ZARDO; MEZZARI, 2004).

\section{CONCLUSÃO}

A partir desta pesquisa constatou-se o predomínio de infecções por Candida spp. em pacientes atendidos pela UTI. A maioria dos isolados foi obtida de amostras de urina e a espécie mais prevalente foi C. albicans. Notou-se também significativo índice de resistência aos antifúngicos utilizados no âmbito hospitalar, principalmente à classe farmacológica dos azólicos. Contudo, os elevados índices de sensibilidade observados frente à anfotericina B e flucitosina mantêm estes fármacos entre as melhores opções terapêuticas nas candidíases complicadas.

Desta forma pôde-se evidenciar que as infecções por Candida spp. ocorrem no ambiente hospitalar principalmente em pacientes debilitados e por leveduras que podem apresentar resistência ou sensibilidade dose-dependente a vários dos antifúngicos utilizados comumente em hospital. Isto aponta a avaliação de sensibilidade como uma estratégia para a seleção do antifúngico e da dose mais adequados, a fim de se alcançar maior sucesso terapêutico principalmente para o tratamento de pacientes críticos.

\section{AGRADECIMENTOS}

Os autores agradecem a disponibilização das amostras pelo Laboratório Brasil, Chapecó (SC).

\section{REFERÊNCIAS}

ALVARES, C. A.; SVIDZINSKI, T. I. E.; CONSOLARO, M. E. L. Candidíase vulvovaginal: fatores predisponentes do hospedeiro e virulência das leveduras. J Bras Patol Med Lab., Rio de Janeiro, v. 43, n. 5, p. 319-327, out. 2007.
AVRELLA, D.; GOULART, L. S. Isolamento de Candida spp. da mucosa oral de pacientes submetidos ao tratamento quimioterápico. RBAC, v. 40, n. 1, p. 205-207, jul. 2008.

BARBEDO, L. S.; SGARBI, D. L. S. Candidíase. DST - J Bras Doenças Sex Transm., v. 22, n. 1, p. 22-38, abr. 2010.

COLOMBO, A. L.; GUIMARÃES, T. A. L. Epidemiologia das Infecções hematogênicas por Candida spp. Rev Soc Bras Med Trop., Uberaba, v. 36, n. 5, p. 599-607, set./ out. 2003.

FIDEL, P. L. JR.; VAZQUEZ, J. A.; SOBEL, J. D. Candida glabrata: Review of Epidemiology, Pathogenesis, and Clinical Disease with Comparison to C. albicans. Clin Microbiol Rev., v. 12, n. 1, p. 80-96, jan. 1999.

FRANÇA, J. C. B.; RIBEIRO, C. E. L.; QUEIROZ-TELLES, F. Candidemia em um hospital terciário brasileiro: incidência, frequência das diferentes espécies, fatores de risco e suscetibilidade aos antifúngicos. Rev Soc Bras Med Trop., Uberaba, v. 41, n. 1, p. 23-28, jan./fev. 2008.

GIOLO, M. P.; SVIDZINSKI, T. I. E. Phisiopathogenesis, epidemioly and laboratory diagnosis of candidemia. $\mathbf{J}$ Bras Patol Med Lab., v. 46, n. 3, p. 225-234, jun. 2010.

HINRICHSEN, S. L.; FALCÃO, É.; VILELLA, T. A. S.; COLOMBO, A. L.; NUCCI, M.; MOURA, L.; RÊGO, L.; LIRA, C.; ALMEIDA, L. Candidemia em hospital terciário do nordeste do Brasil. Rev Soc Bras Med Trop., Uberaba, v. 41, n. 4, p. 394-398. jul./ago. 2008.

MALUCHE, M. E.; SANTOS, J. I. Candida sp. e infecções hospitalares: Aspectos epidemiológicos e laboratoriais. RBAC, v. 40, n.1, p. 65-67, dez. 2008.

MARTINS-DINIZ, J. N.; SILVA, R. A. M.; MIRANDA, E. T.; MENDES-GIANNINI, M. J. S. Monitoramento de fungos anemófilos e de Leveduras em Unidade hospitalar. Rev Saúde Pública, São Paulo, v. 39, n. 3, p. 398-405, jun. 2005.

MENEZES, E. A.; MENDES, L. G.; CUNHA, F. A. Resistência a antifúngicos de Candida tropicalis isoladas no Estado do Ceará. Rev Soc Bras Med Trop., Uberaba, v. 42, n. 3, p. 354-355, maio/jun. 2009. 
NEUFELD, P. M. Caracterização taxonômica e susceptibilidade a antifúngicos de leveduras isoladas de infecção hospitalar. 2009. 256f. Tese (Doutorado em Vigilância Sanitária) - Fundação Oswaldo Cruz, Rio de Janeiro, 2009.

OLIVEIRA, T. M. Infecção urinária hospitalar causada por leveduras do gênero Candida. 2007. 32f. (Especialização em Microbiologia) - Universidade Federal de Minas Gerais, Belo Horizonte, 2007.

PEREIRA, A. P. V. Identificação molecular de candidoses invasivas no Centro Hospitalar Cova da Beira, E.P.E: métodos convencionais vs. métodos moleculares. 2010. 146f. Tese (Mestrado em Ciências Biomédicas) - Instituto de Higiene e Medicina Tropical - Universidade Nova de Lisboa, Lisboa, 2010.

RÖRIG, K. C. O.; COLACITE, J.; ABEGG, M. A. Produção de fatores de virulência in vitro por espécies patogênicas do gênero Candida. Rev Soc Bras Med Trop., Uberaba, v. 42 , n. 2, p. 225-227, mar./abr. 2009.

ROSSI, T.; LOZOVOY, M. A. B.; SILVA, R. V. et al. Interações entre Candida albicans e Hospedeiro. Semina: Ciências Biológicas e da Saúde, Londrina, v. 32, n. 1, p. 15-18, jan.jun. 2011.

SANTANA, D. P.; RIBEIRO, E. L.; MENEZES, A. C. S.; NAVES, P. L. F. Novas abordagens sobre os fatores de virulência de Candida albicans. Rev Ciênc Méd Biol., Salvador, v. 12, n. 2, p. 229-233, maio/ago. 2013.

SILVA, R. F. Capítulo 8: Infecções fúngicas em imunocomprometidos. J Bras Pneumol., São Paulo, v. 36, n. 1, p. 142-147, jan./fev. 2010.

TAMURA, N. K.; NEGRI, M. F. N.; BONASSOLI, L. A.; SVIDZINSKI, T. I. E. Fatores de virulência de Candida spp isoladas de cateteres venosos e mãos de servidores hospitalares. Rev Soc Bras Med Trop., Uberaba, v. 40, n. 1, p. 91-93, jan./fev. 2007.

ZARDO, V.; MEZZARI, A. Os antifúngicos nas infecções por Candida sp. News Lab. v. 63, p.136-146, 2004.
Recebido em: 11 de dezembro de 2014 Aceito em: 17 de março de 2015 